\title{
Synaptonemal complex analysis of an X-autosome translocation-carrier in the domestic pig
}

\author{
DAF Villagomez ${ }^{1}$, M Świtoński ${ }^{2}$ B Singh ${ }^{3}$, KRS Fisher ${ }^{3}$, \\ I Gustavsson ${ }^{1}$, PK Basrur ${ }^{3}$ \\ 1 Swedish University of Agricultural Sciences, \\ Department of Animal Breeding and Genetics, Uppsala, Sweden; \\ ${ }^{2}$ Academy of Agriculture, Department of Genetics and Animals Breeding, \\ Wolyńska 33, 60-637 Poznań, Poland; \\ 3 University of Guelph, Department of Biomedical Sciences, Guelph, Canada
}

(Proceedings of the 9th European Colloquium on Cytogenetics of Domestic Animals; Toulouse--Auzeville, 10-13 July 1990)

synaptonemal complex analysis / $\mathrm{X}$-autosome translocation / domestic pig

Sporadic cases of X-autosome translocations have been found in domestic animals and, recently, Gustavsson et al (1989) reported the first finding of such a translocation in the domestic pig. The present investigation deals with a synaptonemal complex (SC) analysis by electron microscopy of another X-autosome translocation in a sterile male pig, which revealed meiotic arrest at the primary spermatocyte level (Singh, 1990). The translocation occurred proximally in chromosome 14 and terminally in Xp (fig 1a).

In total, 57 spermatocytes in the pachytene stage were investigated using the technique described earlier (Counce and Meyer, 1973). The translocation chromosomes formed 2 bivalents in 51 cells $(89.5 \%)$ in which the axes of the $\mathrm{Y}$ and the $14 \mathrm{q}$ - chromosomes were completely paired into a bivalent configuration similar to that of the normal autosomal bivalents (fig 1b). The bivalent was easy to identify, since it was the smallest SC configuration. The common association with a chromocenter was also easily discernible. The second bivalent, consisting of chromosomes 14 and $\mathrm{Xp}+$, was heteromorphic with axes of unequal size. The longer axis was formed by the $\mathrm{Xp}+$, with the unpaired segment appearing stranded and sometimes folded back and pairing end-to-end with the free end of chromosome 14. Most often ( 42 cells), the axis of chromosome 14 was completely paired with chromosome $\mathrm{Xp}+$, which occasionally ( 3 cells) adjusted its length to become completely aligned with chromosome 14 . This resulted in a remarkable thickness and despiralization of the interstitial part of chromosome $\mathrm{Xp}+$ (fig 1c). In 9 spermatocytes, the 'heterobivalent' showed incomplete synapsis of chromosome 14. A few cells (5 


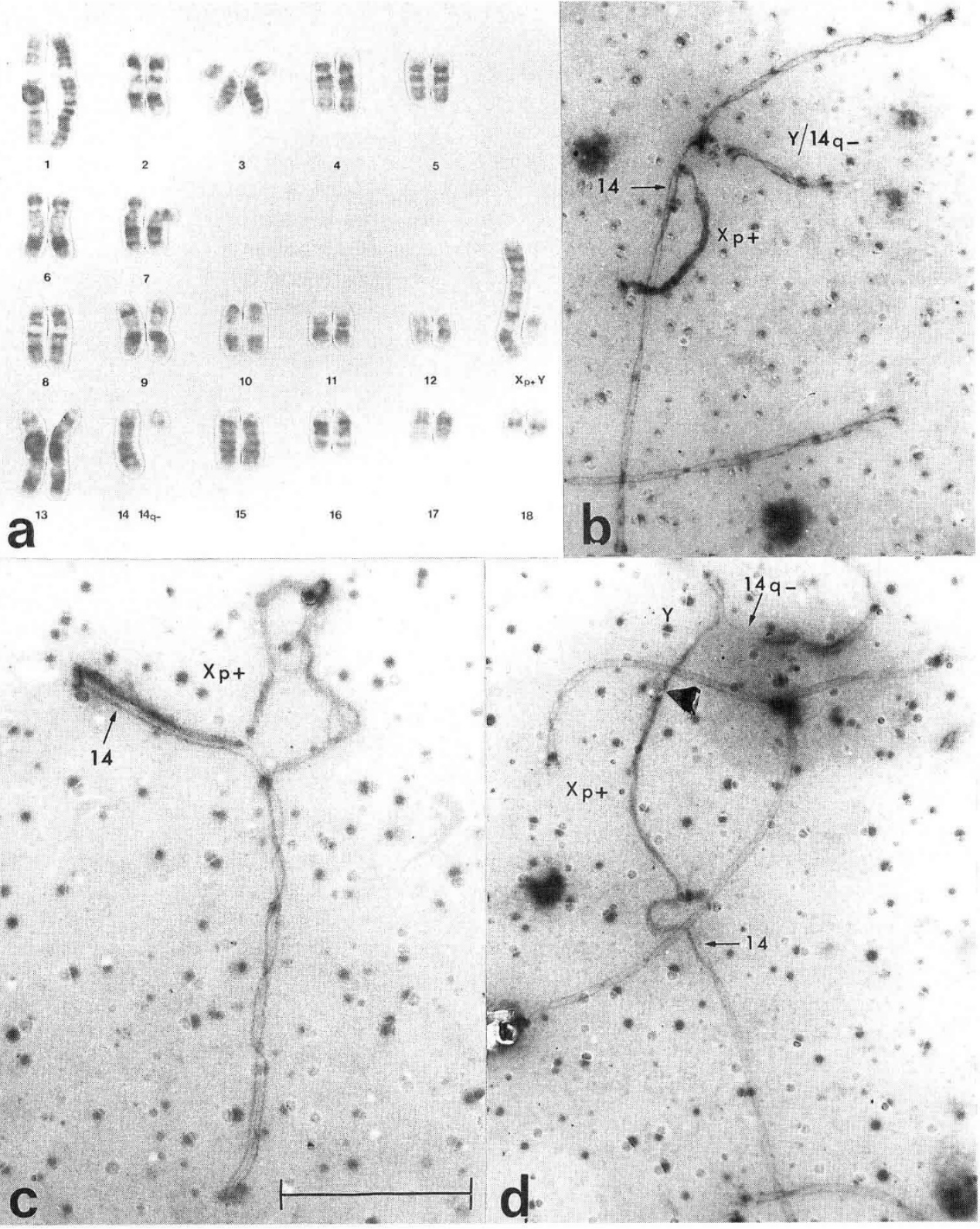

Fig 1. a. GTG-banded karyotype of the $\operatorname{rcp}(\mathrm{Xp}+/ 14 q-)$. b, $\mathbf{c}$ and $\mathbf{d}$. Partial spermatocytes demonstrating translocation configurations. $b$. Heterobivalent with unequal-sized axes formed by $\mathrm{Xp}+$ and 14, and small normal-looking bivalent formed by $\mathrm{Y}$ and $14 \mathrm{q}-$. c. Homologous and heterologous synapses of $\mathrm{Xp}+$ and 14 with partial despiralization of $\mathrm{Xp}+$. d. Open quadrivalent with $\mathrm{Xp}+$ associated end-to-end (arrowhead) with Y. Bar indicates approximately $20 \mu \mathrm{m}$ in $\mathbf{a}, 5.4 \mu \mathrm{m}$ in $\mathbf{b}$ and $\mathbf{d}$ and $3.5 \mu \mathrm{m}$ in $\mathbf{c}$. 
out of 57) exhibited end-to-end associations between the unpaired segment of the $\mathrm{Xp}+$ axis and normal autosomal bivalents and, in 9 cells, chromosome 14 showed such pairings. In a small proportion of cells (10.5\%), the translocation product was an open quadrivalent, formed by the association of either the free segment of $\mathrm{Xp}+$ ( 3 cells), or the proximal segment of chromosome 14 (3 cells), with the axis of either the Y chromosome or chromosome $14 q-$, respectively. Since there is no homology between the unpaired segment of chromosome $\mathrm{Xp}+$ (since it belongs to the $\mathrm{q}$ arm of the $\mathrm{X}$ chromosome) and the $\mathrm{Y}$ chromosome, the association observed should be non-homologous (fig 1d). There was only one quadrivalent in which pairing of chromosome 14 with the centromeric end of $14 q-$ led to central element formation.

Contrary to the theoretical expectations, the present aberration most frequently formed 2 bivalents instead of a quadrivalent. Nevertheless, unequivocal identification of the pairing region of the sex chromosomes could be made by correlating the findings from the somatic chromosome analysis with those of the synaptic analysis of the translocation. Since the derivative chromosome $14 q-$ always paired with the $\mathrm{Y}$ chromosome and the breakpoint in $\mathrm{X}$ was observed distally in the short arm, it can be concluded that the $\mathrm{X}$ and $\mathrm{Y}$ pairing segment lies distally in the $\mathrm{Xp}$ arm. At early pachytene, the pairing of the pig sex chromosomes often includes the total Y (Schwarzacher et al, 1984). In the present translocation, the Y chromosome also synapsed completely with chromosome $14 \mathrm{q}-$, which had obtained its terminal part from the $\mathrm{Xp}$ arm and conserved the centromeric region of chromosome 14 . The bivalent, formed by the $\mathrm{Y}$ chromosome and derivative $14 \mathrm{q}-$, should be considered a result of homologous and non-homologous pairing. The same should be true when chromosome 14 was completely paired with chromosome $\mathrm{Xp}+$. A similar heterologous synapsis was observed in heteromorphic bivalents of $2 \mathrm{X}$-autosome translocations (R5 and R6) in male mice (Ashley, 1983). In Gallus domesticus also, cytological evidence suggests that ZW gonosomes undergo non-homologous pairing in Z-autosome translocations (Solari et al, 1988). The formation of bivalents may be due to pairing competition. When the $\mathrm{Xp}+$ and the $\mathrm{Y}$ chromosomes are completely paired with chromosome 14 and derivative $14 q-$, respectively, they probably prevent the pairing of the latter two chromosomes and lead to a high frequency of bivalent formation. Although in some spermatocytes (9 out of 57 cells) the heterobivalent exhibited unpaired segments of chromosome 14, the Y chromosome was always completely paired with chromosome $14 q-$. Finally, it is clear that the pairing is non-homologous in the open quadrivalents formed by the association of the $\mathrm{Xp}+$ axis with the axes of the $\mathrm{Y}$ and chromosome $14 \mathrm{q}-$.

There is only one previous report of an $\mathrm{X}$-autosome reciprocal translocation in the domestic pig (Gustavsson et al, 1989). In that case as well as in the present translocation, the male was sterile, which is a common phenomenon in this type of translocation in man and mouse (reviewed by Mattei et al, 1982; Searle, 1982). According to Lifschytz and Lindsley (1972), the inactivation of the X chromosome is essential for normal spermatogenesis, which means that autosomal material translocated to the $\mathrm{X}$ chromosome interferes with $\mathrm{X}$ chromosome inactivation. This hypothesis has also been supported by the observation that the unpaired chromosome segments in male-sterile autosome-autosome rearrangements in man and mouse are frequently associated with the sex bivalent (eg, Chandley, 1982). 


\section{REFERENCES}

Ashley T (1983) Nonhomologous synapsis of the sex chromosomes in the heteromorphic bivalents of two X-7 translocations in male mice: R5 and R6. Chromosoma 88, 178-183

Chandley AC (1982) Normal and abnormal meiosis in man and other mammals. In: Serono Clinical Colloquium on Reproduction (Crosignani PG et al, eds) Academic Press/Grune \& Stratton

Counce SJ, Meyer GF (1973) Differentiation of the synaptonemal complex and the kinetochore in Locusta spermatocytes studied by whole mount electron microscopy. Chromosoma 44, 231-253

Gustavsson I, Świtoński M, Iannuzzi L, Plöen L, Larsson K (1989) Banding studies and synaptonemal complex analysis of an $\mathrm{X}$-autosome translocation in the domestic pig. Cytogenet Cell Genet 50, 188-194

Lifschytz E, Lindsley DL (1972) The role of X-chromosome inactivation during spermatogenesis. Proc Natl Acad Sci USA 69, 182-186

Mattei MG, Mattei JF, Ayne S, Giraud F (1982) X-autosome translocations: cytogenetic characteristics and their consequences. Hum Genet 61, 295-309

Schwarzacher T, Mayr B, Schweizer D (1984) Heterochromatin and nucleolus organizer region behavior at male pachytene of Sus scrofa domestica. Chromosoma 91, 12-19

Searle AG (1982) The genetics of sterility in the mouse. In: Serono Clinical Colloquium on Reproduction (Crosignani PG et al, eds) Academic Press/Grune \& Stratton

Singh B (1990) An X-autosome translocation in the pig: characterization and impact on fertility. Master's Thesis, University of Guelph, Guelph, Ontario, Canada

Solari AJ, Fechheimer NS, Bitgood JJ (1988) Pairing of ZW gonosomes and the localized recombination nodule in two Z-autosome translocations in Gallus domesticus. Cytogenet Cell Genet 18, 130-136 